\title{
Numerical Simulation of the Viscohyperelastic Behaviour of PET near the Glass Transition Temperature
}

\author{
Yun Mei Luo ${ }^{1, a}$, Luc Chevalier ${ }^{1, b}$ and Eric Monteiro ${ }^{1, c}$ \\ ${ }^{1}$ Université Paris-Est, Laboratoire Modélisation et Simulation Multi Echelle, MSME UMR 8208 \\ CNRS, 5 bd Descartes, 77545 Marne-la-Vallée, France \\ ayunmei.luo@univ-paris-est.fr, bluc.chevalier@univ-paris-est.fr, ${ }^{\mathrm{c} e r i c . m o n t e i r o @ ~ u n i v-p a r i s-e s t . f r ~}$
}

Keywords: Numerical simulation, viscohyperelastic, nonlinear behaviour, thermo-mechanical equation

\begin{abstract}
The presentation deals with the non linear strongly elastic and viscous behaviour of poly ethylene terephthalate near the glass transition temperature and biaxially stretched at high strain rates representative of the injection stretch blow moulding process. A non linear visco-hyperelastic model identified from the experimental results of the equi-biaxial tension test is implemented into a finite element code developed in the Matlab environment. The thermal behaviour modelling, identification and simulation has also been managed. The model was used to simulate the $2 \mathrm{D}$ plane stress case and then was applied to a 2D axi-symmetric case. Both simulations are compared with equi biaxial testing. The final goal of this work is to perform the free blowing simulation to compare with experimental data. Therefore, we should solve an iterative procedure for a thermo-mechanical equation. At each time step, the proposed visco-hyperelastic model is used for the mechanical part, and a classical heat transfer equation is discretized for the thermal part. All mechanical parameters are reactualized from the temperature field.
\end{abstract}

\section{Introduction}

The injection stretch blow moulding (ISBM) process which is managed at a temperature near or slightly above the glass transition temperature Tg involves multiaxial large strains at high strain rate of the polyethylene terephthalate material (PET). During the ISBM process, the PET behaviour exhibits a highly elasticity, a strain hardening effect and a strong viscous and temperature dependency. Therefore, much research has been conducted on the rheological behaviour of PET. Essentially, the viscoelastic model which take into account the strain hardening and strain rate effects have been widely used for ISBM process in literature. Inspired from Buckley model [1], we proposed a non linear incompressible visco-hyperelastic model to model the complex constitutive behaviour of PET [2]. Based on the experimental results of the equi-biaxial tension test [3], we identified the properties of this visco-hyperelastic behaviour. In this work, we implemented the proposed model into a finite element code developed with Matlab. Moreover, the thermal behaviour modelling, identification and simulation has also been managed.

First, a numerical simulation of 2D plane stress case has been performed involving 2 fields (global velocity V and elastic Cauchy Green tensor Be). Rectangular finite elements with quadratic and linear interpolations have been employed for velocity and the elastic left Cauchy Green tensor. Degree of interpolation has been tested for all possible combinations to test the Ladyzenskaia-Babushka-Brezzi (LBB) like condition [4]. Second, an axial symmetric formulation involving 4 fields (global velocity $\mathrm{V}$, Lagrange multiplier $\mathrm{p}$ associated with the global incompressibility condition, and multiplier $\mathrm{q}$ associated with the incompressibility of the elastic part) has been performed using rectangular elements. Both simulations are compared with equi-biaxial testing in order to reproduce the strain hardening effect and the self-heating observed.

In order to accurately simulate the ISBM process, a thermo-mechanical model was used. Mechanical and thermal equilibrium equations are fully non linear and solved together with implicit schemes on the current deformed configuration, which is updated at each time step. Biaxial 
characterization tests were used to manage the identification of the model parameters, in order to simulate the ISBM process.

\section{Modelling the Viscohyperelastic Behaviour of PET}

Inspired from Figiel and Buckley [1], we proposed a non linear incompressible visco-hyperelastic model with nonlinear forms for both elastic and viscous parts to represent the mechanical behaviour.

$$
\left\{\begin{array}{l}
\underline{\hat{\sigma}}=2 G \underline{\underline{\hat{\varepsilon}_{e}}} \\
\underline{\underline{\underline{\hat{\sigma}}}}=2 \eta \underline{\underline{D_{v}}}
\end{array} \quad \text { and } \quad \underline{\underline{\sigma}}=2 \eta_{N} \underline{\underline{D}}+\underline{\underline{\hat{\sigma}}}-p \underline{\underline{I}}-q \underline{\underline{I}}\right.
$$

where $\underline{\underline{\sigma}}$ is the Cauchy stress tensor, $\underline{\underline{D}}_{v}$ is the symmetric part of the viscous velocity gradient, the subscript " $\wedge$ " denotes the deviatoric part of the tensor, $\eta_{N}$ is the small value of the viscosity of the Newtonian branch of the Zener like modelling used in order to solve the ill-conditioned problem, $\underline{\underline{\varepsilon}}_{\underline{e}}$ is the elastic part of the Eulerian strain measure defined by:

$$
\left.\underline{\underline{\varepsilon_{e}}}=\frac{1}{2} \underline{\underline{\left(B_{e}\right.}}-\underline{\underline{I}}\right)
$$

Where $\underline{\underline{B}}_{\underline{e}}$ is the elastic left Cauchy Green tensor. $p$ is a Lagrange multiplier associated to the global incompressibility condition, and $q$ is the multiplier associated to the incompressibility of the elastic part. Since the elastic and global parts are incompressible, the viscous part is supposed to be also incompressible:

$$
\operatorname{det} \underline{\underline{B_{e}}}=1, \quad \operatorname{div} \vec{V}=\operatorname{trace} \underline{\underline{D}}=0, \quad \operatorname{div} \vec{V}_{v}=\operatorname{trace} \underline{\underline{D_{v}}}=0
$$

The assumption of an additive decomposition of elastic and viscous velocity gradient is adopted to describe the kinematic structure of this model:

$$
\underline{\underline{D}}=\underline{\underline{D_{e}}}+\underline{\underline{D_{v}}}
$$

Combining equations, 1, 2 and 4 in the Oldroyd derivation of the elastic left Cauchy-Green tensor leads to:

$$
\frac{\delta \underline{\underline{B_{e}}}}{\delta t}+\frac{G}{\eta} \underline{\underline{B_{e}}} \underline{\underline{\hat{B}_{e}}}=0
$$

where $G$ is the elastic shear modulus and $\eta$ is the ratio of the viscosity. In order to represent the strain hardening and strain rate effect and temperature dependency, we choose two rheological functions for elastic and viscous parts: $G\left(\varepsilon_{e}\right)$ and $\eta\left(\varepsilon_{v}, \overline{\dot{\varepsilon}_{v}}, \mathrm{~T}\right)$.

$$
\left\{\begin{array}{l}
\underline{\underline{\hat{\sigma}}}=2 G\left(\varepsilon_{e}\right) \underline{\underline{\underline{\hat{\varepsilon}_{e}}}} \\
\underline{\underline{\hat{\sigma}}}=2 \eta\left(\varepsilon_{v}, \dot{\bar{\varepsilon}}_{v}, T\right) \underline{\underline{D_{v}}}
\end{array} \quad \text { and } \quad \underline{\underline{\sigma}}=2 \eta_{N} \underline{\underline{D}}+\underline{\underline{\hat{\sigma}}}-p \underline{\underline{I}}-q \underline{\underline{I}}\right.
$$

where $\varepsilon_{e}$ is the equivalent elastic strain and $\overline{\dot{\varepsilon}_{v}}$ is the equivalent viscous strain rate.

\section{Identification of the Material's Properties}

One can first identify the initial shear modulus $\mathrm{G}_{0}$ from the initial slope of the global experimental strain-stress curves which were carried out by Menary et al. [3].

Table 1. The numerical value of $\mathrm{G}_{0}$

\begin{tabular}{l|c|c|c|c|c}
\hline Strain Rate $(/ \mathrm{s})$ & 1 & 2 & 4 & 8 & 16 \\
\hline $\mathrm{G}_{0}(\mathrm{MPa})$ & 7.2 & 8.1 & 7.7 & 7.9 & 8.9 \\
\hline $\mathrm{Min} \mathrm{G}_{0}(\mathrm{MPa})$ & \multicolumn{5}{|c}{7.2} \\
\hline $\mathrm{Max} \mathrm{G}_{0}(\mathrm{MPa})$ & \multicolumn{7}{c}{8.9} \\
\hline
\end{tabular}


As they are conducted at constant nominal strain rate, during the biaxial tests, the global strain rate decreases versus time. The shear modulus $\mathrm{G}_{0}$ cannot remain constant because the elastic strain rate increases too much and may exceed the global strain rate. Therefore, we consider a Hart-Smith like model to represent the elastic part:

$$
G=G_{0} \exp \left(\Lambda\left(I_{1}-3\right)^{2}\right), I_{1}=\operatorname{trace}\left(B_{e}\right)
$$

For the non-linear viscous part of the model we follow the same method as in Cosson and Chevalier [5] to represent macroscopically the strain hardening effect. Instead we choose a Carreau type law instead of the power law in the viscous model:

$$
\eta=\eta_{0} h\left(\overline{\varepsilon_{v}}\right) \cdot f\left(\overline{\dot{\varepsilon}}_{v}\right), f\left(\overline{\dot{\varepsilon}}_{v}\right)=\left(1+\left(\lambda\left(\overline{\dot{\varepsilon}}_{v} / \dot{\varepsilon}_{r e f}\right)\right)^{a}\right)^{\frac{m-1}{a}}
$$

where $\dot{\varepsilon}_{\text {ref }}$ is a reference strain rate that can be taken equal to $1 \mathrm{~s}^{-1}$. The strain hardening effect is related to the $h$ function which increases continuously with $\bar{\varepsilon}_{v}$. We detailed the identification procedure for the $h$ function in [3]. The strain hardening effect is influenced by the temperature. Therefore, $h$ is a function of $T$ also:

$$
\eta_{0} h\left(\overline{\varepsilon_{v}}, T\right)=\frac{\eta_{0}(T) \cdot\left(1-\exp \left(-K \overline{\varepsilon_{v}}\right)\right)}{\left(1-\overline{\varepsilon_{v}} / \varepsilon_{v \lim }(T)\right)^{N}}
$$

Parameters $K$ and $N$ do not vary much with the temperature; at the contrary, variables $\eta_{0}(T)$ and $\varepsilon_{v l i m}(T)$ show a significant dependence on temperature. We choose the Williams-Landel-Ferry (WLF) model for the evolution of $\eta_{0}(T)$ :

$$
\ln \left(a_{T}\right)=\frac{-C_{1}\left(T-T_{r e f}\right)}{C_{2}+T-T_{r e f}}
$$

where $C_{1}$ and $C_{2}$ are the WLF parameters, $T_{r e f}=90^{\circ} \mathrm{C}$. We propose the evolution of $\varepsilon_{v l i m}(T)$ in the following way:

$$
\varepsilon_{v \lim }=\varepsilon_{v \lim \_r e f}\left(1+\frac{B_{1}\left(T_{r e f}-T\right)}{\left(T-B_{2}\right)}\right)
$$

\begin{tabular}{|c|c|c|c|c|c|c|}
\hline \multirow{2}{*}{\multicolumn{2}{|c|}{$G\left(\varepsilon_{e}\right)$}} & \multirow{2}{*}{\multicolumn{3}{|c|}{$\frac{\mathrm{G}_{0}}{8 \mathrm{Mna}}$}} & \multicolumn{2}{|c|}{$\Lambda$} \\
\hline & & & & & \multicolumn{2}{|c|}{0.001} \\
\hline \multirow{6}{*}{$\eta\left(\varepsilon_{v}, \overline{\dot{\varepsilon}_{v}}, \mathrm{~T}\right)$} & \multirow{2}{*}{$f\left(\overline{\dot{\varepsilon}_{v}}\right)$} & \multirow{2}{*}{\multicolumn{2}{|c|}{$\frac{\lambda}{9.91}$}} & $\mathrm{a}$ & \multicolumn{2}{|r|}{$\mathrm{m}$} \\
\hline & & & & 2 & \multicolumn{2}{|r|}{0.2} \\
\hline & \multirow[t]{2}{*}{$h\left(\overline{\varepsilon_{v}}\right)$} & $\eta_{0}$ & $K$ & $h_{0}$ & $N$ & $\mathcal{E}_{\text {Vlim_ref }}$ \\
\hline & & 8.4 Mpa.s & 3.2 & -0.21 & 0.42 & 1.83 \\
\hline & \multirow{2}{*}{$\begin{array}{c}\eta_{0}(T), \\
\varepsilon_{\text {vlim }}(T)\end{array}$} & $C_{1}$ & \multicolumn{2}{|c|}{$C_{2}$} & & $B_{2}$ \\
\hline & & 1.88 & \multicolumn{2}{|c|}{$25.81^{\circ} \mathrm{C}$} & .07 & $111.88^{\circ} \mathrm{C}$ \\
\hline
\end{tabular}

where $\varepsilon_{v \lim _{-} \text {ref }}=\varepsilon_{v{\lim \_90^{\circ} \mathrm{C}}^{\circ}}$. Finally, the characteristics of the PET for these visco-hyperelastic model expressions to represent conveniently the experimental are:

Table 2. The characteristics of the PET

\section{Numerical Implementation of the Model}

The four-field approach is adopted for the numerical implementation. Some standard manipulations lead to the following weak form:

$$
\begin{aligned}
& \int_{\Omega} \underline{\underline{D}}^{*}: G \underline{\underline{\hat{B}_{e}}} d \Omega+\int_{\Omega} \underline{\underline{D}}^{*}: 2 \eta_{n} \underline{\underline{D}} d \Omega-\int_{\Omega} \underline{\underline{D}}^{*}: \underline{\underline{I}}\left(p_{e}+q\right) d \Omega-\int_{\partial \Omega_{F}} \underline{V}^{*} \underline{F}^{d} d S=0 \\
& \int_{\Omega} p^{*} \underline{\underline{I}}: \underline{\underline{D}} d \Omega=0 \\
& \int_{\Omega} q^{*}\left(\operatorname{det}\left(\underline{\underline{B_{e}}}\right)-1\right) d \Omega=0
\end{aligned}
$$




$$
\int_{\Omega} \underline{\underline{B_{e}}}{ }^{*}: \underline{\underline{\dot{B}_{e}}} d \Omega+2 \int_{\Omega} \underline{\underline{B_{e}}}: \underline{\underline{B_{e}}} \underline{\underline{\Omega}} d \Omega-2 \int_{\Omega} \underline{\underline{B_{e}}}: \underline{\underline{B_{e}}} \underline{\underline{\underline{D}}} d \Omega+\int_{\Omega} \underline{\underline{B_{e}}}{ }^{*}: \frac{G}{\eta} \underline{\underline{B_{e}}} \underline{\underline{\hat{B}_{e}}} d \Omega=0
$$

Where the superscript $*$ denotes test quantities and $\underline{F}^{d}$ is the prescribed traction field over the boundary $\partial \Omega_{F}$.

To solve this nonlinear problem (finite elastic displacements, non constant shear modulus $G$ and viscosity $\eta$ ) an iterative procedure (e.g. Newton-Raphson) must be used. A consistent linearization is achieved with the help of the Gâteaux operator. In the equi-biaxial elongation plane stress case, before deriving the weak form, we can establish a relation between the pressures and the velocity and the elastic left Cauchy Green tensor:

$$
\sigma_{33}=0 \Rightarrow p+q=-2 \eta_{N}\left(D_{11}+D_{22}\right)+\frac{G}{3}\left(\frac{2}{B_{11} B_{22}-B_{12}^{2}}-B_{11}-B_{22}\right)
$$

Therefore, the searched solution is a mixed velocity $\underline{V}$ and $\underline{\underline{B}}_{e}$ formulation and the weak form over the entire volume $\Omega$ is given by:

$$
\begin{aligned}
& 2 \eta_{N} \int_{\Omega} \underline{\underline{D}}^{*}: \underline{\underline{D}} d \Omega+G \int_{\Omega} \underline{\underline{D}}^{*}: \underline{\underline{\hat{B}_{e}}} d \Omega+2 \eta_{N} \int_{\Omega} \underline{\underline{D}}^{*}: \underline{\underline{I}}\left(D_{11}+D_{22}\right) d \Omega \\
& -\frac{G}{3} \int_{\Omega} \underline{\underline{D}}^{*}: \underline{\underline{I}} \frac{1}{B_{e 11} B_{e 22}-B_{e 12}{ }^{2}} d \Omega-\int_{\partial \Omega_{F}} \underline{V}^{*} \underline{F}^{d} d S=0 ; \\
& \int_{\Omega} \underline{B_{e}^{*}}:\left(\frac{\delta \underline{\underline{B_{e}}}}{\delta t}+\frac{G}{\eta} \underline{\underline{B_{e}}} \underline{\underline{\hat{B}_{e}}}\right) d \Omega=0
\end{aligned}
$$

\section{Simulation the Biaxial Plan Stress Testing}

The domain $\Omega$ is approximated by a set of 8 -nodes isoparametric rectangles. In the case of the classical incompressible problem (a mixed velocity and pressure formulation), the finite element calculations are not stable, some of them showing pressure oscillations if velocity and pressure spaces are not chosen carefully. To be stable, a mixed formulation must verify consistency. The well-known inf-sup condition or the LBB condition [4] guaranties the stability of a finite element velocity pressure calculation. In our two-field approaches $\underline{V}$ and $\underline{\underline{B}}_{\mathrm{e}}$ case, we tested different interpolations to obtain accurate solutions. We choose the different shape function for the convergence test: VLBL is the linear interpolation for both $\underline{V}$ and $\underline{\underline{B}}_{\mathrm{e}}$; VLBQ is the linear interpolation for $\underline{V}$ and quadratic for $\underline{\underline{B}}_{\mathrm{e}}$; VQBL is the quadratic interpolation for $\underline{V}$ and linear for $\underline{\underline{B}}_{\mathrm{e}}$; VQBQ is the quadratic interpolation for both $\underline{V}$ and $\underline{\underline{B}}$. The error is the comparison between numerical and analytical results of stress in the equi-biaxial elongation case.

$$
\text { error }=\frac{\left|\sigma_{\text {num }}-\sigma_{\text {ana }}\right|}{\left|\sigma_{\text {ana }}\right|}
$$

The errors should decrease when the size of the element $h$ decrease.

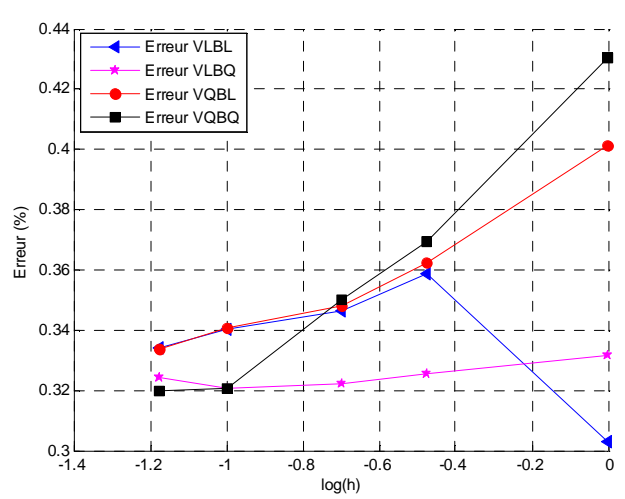

Figure 1. The errors versus the size of the element $h$ 
We implement the proprieties identified in the numerical simulation for the best choice: interpolation for velocity is quadratic and linear for $\underline{\underline{B}}_{\mathrm{e}}$.

Figures $2 \mathrm{a}$ and $2 \mathrm{~b}$ show a substantially good representation of the experimental results. The mean difference does not exceed $10 \%$.

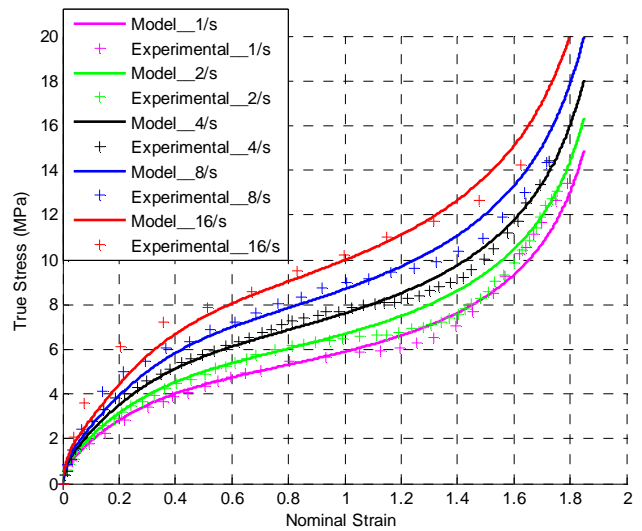

(a)

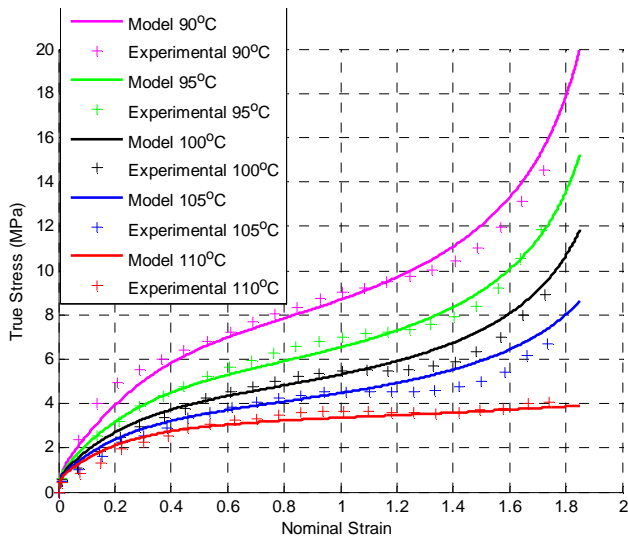

Figure 2. (a) The experimental data (+) and the finite elements results of the visco-elastic model (lines) at $90^{\circ} \mathrm{C}$ under different strain rates; (b) The experimental data $(+)$ and the finite elements results of the visco-elastic model (lines) at $8 \mathrm{~s}^{-1}$ under different temperatures.

\section{Simulation of a Thermo-mechanical Model}

The numerical simulation of the stretch/blow moulding of the proposed visco-hyperelastic mode consists in solving the following set of thermo-mechanical equations on the domain $\Omega$.

The mechanical part is written in Eq. 14 in the plane stress case and the heat part is:

$$
\left\{\begin{array}{l}
\rho c \dot{T}-k \Delta T=\underline{\sigma}: \underline{\underline{D}} \\
k \underline{\nabla} T \cdot \underline{n}=-h_{c}\left(T-T_{\infty}\right) \quad \text { on } \quad \partial \Omega_{q} \\
T=T_{0} \quad \text { at } \quad t=0
\end{array}\right.
$$

where: $\rho$ the mass density, $c$ the specific heat capacity and $k$ is the material's conductivity, $h_{c}$ is the heat transfer coefficient.

Table 3. The value of thermal proprieties

\begin{tabular}{l|c|c|c|c}
\hline Parameters & $\rho\left(\mathrm{kg} / \mathrm{m}^{3}\right)$ & $c(\mathrm{~J} / \mathrm{kg} . \mathrm{K})$ & $k(\mathrm{~W} / \mathrm{m} . \mathrm{K})$ & $h_{c}(\mathrm{~W} / \mathrm{m} 2 . \mathrm{K})$ \\
\hline Values & 1344.6 & 1879.8 & 0.1022 & 28.59 \\
\hline
\end{tabular}

$\partial \Omega_{q}$ is the union of the top and bottom face of the specimen. No thermal exchange is assumed between the specimen and the grips. To be consistent with the plan stress assumption, $T$ is chosen as a function of the plan coordinates $x, y$ and time. Consequently, the weak form writes:

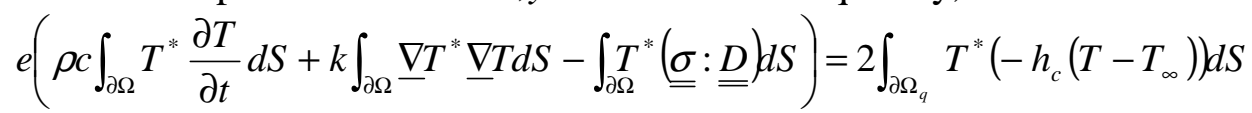

Using an iterative procedure, at each time step, mechanical and temperature balance equations are solved together on the current deformed configuration. Then, the geometry is updated.

Figure 3 shows, that stresses obtained from this thermo-mechanical simulation are lower than the experimental data. Because the temperature increases of about $6^{\circ} \mathrm{C}$ during the biaxial elongation, the self heating effect affects the mechanical properties, especially the viscosity which decreases. Consequently, the parameters identified assuming an isothermal elongation must be modified to take into account this. 


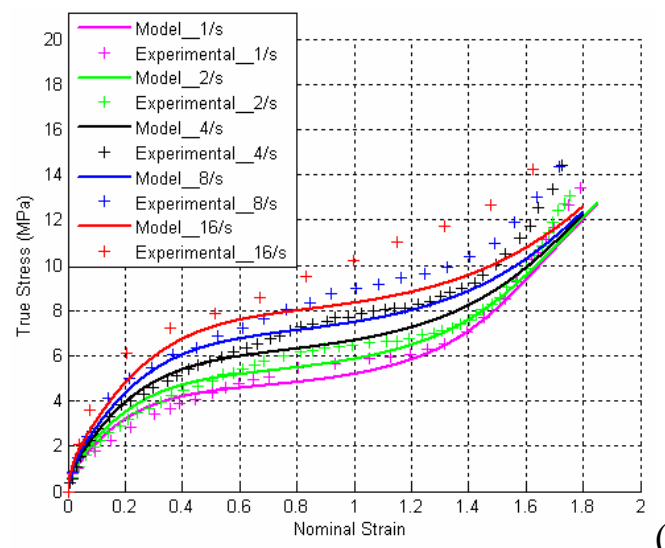

(a)
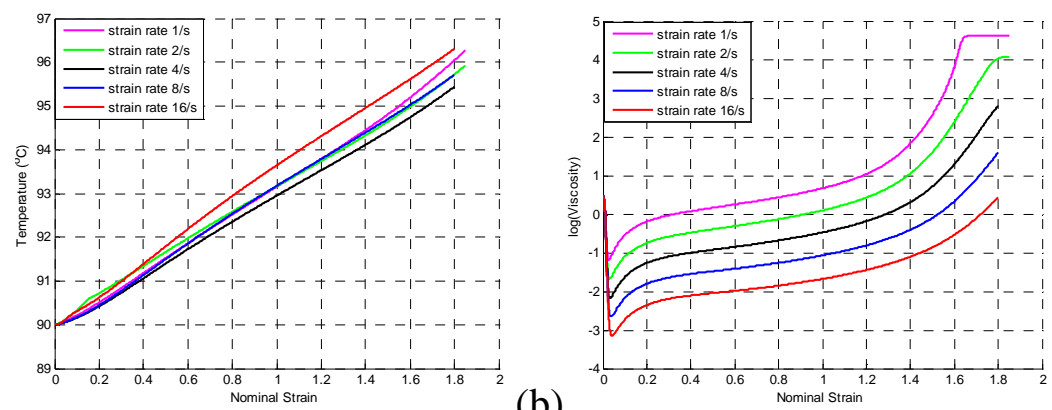

(b)

(c)

Figure 3. (a) The experimental data (the points) and the thermo-mechanical results; (b) The evolution of temperature under different strain rates; $(c)$ The variation of viscosity with temperature under different strain rates.

\section{Conclusions}

A visco-hyperelastic model identified from the equi biaxial tests performed at conditions close to ISBM process strain rate and temperature was implemented for numerical simulations. This finite elements model was used to simulate the plane stress test. It reproduces successfully the experimental results and can be used to simulate uniaxial or sequential biaxial tests to predict the PET behaviour.

Thermal effects have also been identified and we carried out non-isothermal visco-hyperelastic simulations in order to compare with the experimental data.

The parameters identified in the proposed model have to be adjusted because the self heating effect is not negligible and has an important effect on the viscous part of the model. Therefore, in further works, after this adjustment, we intend to implement an axi-symmetric version of the visco-hyperelastic model coupled to temperature in order to simulate accurately the ISBM process.

\section{References}

[1] L. Figiel, C.P. Buckley. On the modeling of highly elastic flows of amorphous thermoplastic. International Journal of Non-linear Mechanics, 44, 389-395, (2009).

[2] Luo Y.M., Chevalier L., Monteiro E., Identification of a Visco-Elastic Model for PET Near Tg Based on Uni and Biaxial Results, The 14th International ESAFORM Conference on Material Forming, Queen's University, Belfast, Irlande du Nord, April 27-29, 2011.

[3] G.H. Menary, C.W. Tan, E.M.A. Harkin-Jones, C.G. Armstrong, P.J. MartinBiaxial Deformation of PET at Conditions Applicable to the Stretch Blow Molding Process. Polymer Engineering \& Science, in press.

[4] F. Brezzi, M. Fortin, Mixed and hybrid Finite Element Methods, Springer :New York, 1991.

[5] B. Cosson, L. Chevalier, J. Yvonnet. Optimization of the thickness of PET bottles during stretch blow molding by using a mesh-free (numerical) method. International Polymer Processing, Volume 24, Issue 3, Pages 223-233, (2009). 